\title{
ON THE COMPATIBILITY OF ALGEBRAIC EQUATIONS WITH EXTENSIONS ${ }^{\dagger}$
}

\author{
HARALD HULE and WINFRIED B. MÜLLER \\ (Received 13 May 1975; revised 26 August 1975)
}

\begin{abstract}
In this paper we investigate the following problem proposed by Lausch and Nöbauer: Let $\mathcal{B}$ be a variety of universal algebras, $B$ an algebra of $\mathfrak{B}$ and $A$ a subalgebra of $B$. If a system of algebraic equations over $A$ is solvable in $\mathfrak{B}$, is it then solvable over $B$ ? We show that the answer is affirmative in certain varieties but negative in the general case.
\end{abstract}

\section{Introduction}

Let $A$ be a universal algebra in the variety $\mathfrak{B}$ and $X=\left\{x_{1}, \cdots, x_{n}\right\}$ a set of indeterminates. An algebra $B$ which contains $A$ as a subalgebra will be called an extension of $A$. If $B \in \mathfrak{B}$, then we call it a $\mathfrak{B}$-extension of $A$. Let $W(A, X)$ be the word algebra in $A \cup X$ as defined by Lausch and Nöbauer (1973). Every $p \in W(A, X)$ induces in a natural way an $n$-ary polynomial function on any extension of $A$.

An algebraic equation in $X$ over $A$ is a pair $(p, q)$ with $p, q \in W(A, X)$, written usually in the form " $p=q$ ". Let $B$ be a $\mathfrak{B}$-extension of $A$. Then an $\boldsymbol{n}$-tuple $\left(b_{1}, \cdots, b_{n}\right)$ of elements of $B$ is called a solution of the equation " $p=q$ " if $p\left(b_{1}, \cdots, b_{n}\right)=q\left(b_{1}, \cdots, b_{n}\right)$ is valid in $B$, where $p\left(b_{1}, \cdots, b_{n}\right)$ means of course the image of $\left(b_{1}, \cdots, b_{n}\right)$ under the polynomial function induced by $p$. A system of algebraic equations over $A\left\langle p_{i}=q_{i}\right\rangle(i \in I)$ is called "solvable over $(A, \mathfrak{B})$ " if there exist a $\mathfrak{B}$-extension $B$ of $A$ and an $n$-tuple $\left(b_{1}, \cdots, b_{n}\right) \in B^{n}$ which satisfies simultaneously the equations of the system.

Systems of algebraic equations have been studied by Hule (1970) and Lausch and Nöbauer (1973). As we are only interested in solutions lying in $\mathfrak{B}$-extensions of $A$, we can identify the words which induce the same polynomial

${ }^{+}$We want to thank the referee of the Journal of the Australian Mathematical Society whose suggestions were essential for the elaboration of the final version. 
functions on every $\mathfrak{B}$-extension of $A$. The classes of equivalent words in this sense are called elements of the "polynomial algebra" $A(X, \mathfrak{B})$ (in fact, $A(X, \mathfrak{B})$ is isomorphic to the classical polynomial ring $A\left[x_{1}, \cdots, x_{n}\right]$ if $\mathfrak{B}$ is the variety of commutative rings with identity), and consequently an algebraic equation over $(A, \mathfrak{B})$ is defined as an expression " $p=q$ " with $p, q \in A(X, \mathfrak{B})$.

Lausch and Nöbauer (1973) proposed the following problem: If $(S)$ is a system of algebraic equations solvable over $(A, \mathfrak{B})$ and $B$ is a $\mathfrak{B}$-extension of $A$, is then $(S)$ also solvable over $(B, \mathfrak{B})$ ? We will show that the answer is affirmative in certain varieties, but negative in the general case. A variety $B$ will be called "solutionally compatible" if for any $A \in \mathfrak{B}$ and any $\mathfrak{B}$-extension $B$ of $A$, the solvability of a system of equations over $(A, \mathfrak{B})$ implies the solvability over $(B, \mathfrak{B})$.

\section{Solutionally compatible varieties}

We shall give a sufficient condition for solutional compatibility. For this we need the following definition: A variety $\mathfrak{B}$ satisfies (AP) if and only if for any two $\mathfrak{B}$-extensions $B$ and $C$ of an algebra $A \in \mathfrak{B}$ there exists a $\mathfrak{B}$-extension $D$ of $B$ which contains a subalgebra $C^{\prime}$ such that $A \subseteq C^{\prime}$ and there exists an isomorphism between $C$ and $C^{\prime}$ which fixes the elements of $A$.

It is easy to verify that (AP) is equivalent to the Amalgamation Property as defined by Jónsson (1961).

Lemma. If a variety $\mathfrak{B}$ satisfies $(A P)$ then it is solutionally compatible.

Proof. Suppose that (AP) holds in $\mathfrak{B}$. Let $B$ be a $\mathfrak{B}$-extension of $A$ and $(S)$ a system of equations solvable over $(A, \mathfrak{B})$. Then there exists a solution $\left(c_{1}, \cdots, c_{n}\right)$ of $(S), c_{1}, \cdots, c_{n} \in C$ for some $\mathfrak{B}$-extension $C$ of $A$. By (AP) we can find a $\mathfrak{B}$-extension $D$ of $B$ with a subalgebra $C^{\prime}$ and an isomorphism $\phi: C \rightarrow C^{\prime}$ which fixes $A$. Then $\left(\phi c_{1}, \cdots, \phi c_{n}\right) \in D^{n}$ is obviously a solution of $(S)$, showing that $(S)$ is solvable over $(B, \mathfrak{B})$.

Corollary. Any variety defined by the empty set of laws, the varieties of groups, of abelian groups, of lattices, of distributive lattices, of distributive lattices with 0 and 1 and the variety of Boolean algebras are solutionally compatible.

Proof. It is sufficient to observe that these varieties satisfy (AP). This is a well-known fact for the varieties of groups and of abelian groups. For the varieties of lattices (AP) has been proved by Jónsson (1956). A proof of (AP) for distributive lattices, distributive lattices with 0 and 1 and Boolean algebras is found in Grätzer (1971). Jónsson (1956) proved (AP) also for the variety of groupoids. An obvious modification of his argument shows that (AP) holds in any variety defined by the empty set of laws. 


\section{Varieties which are not solutionally compatible}

THEOREM. The varieties of semigroups and of rings, of commutative semigroups and of commutative rings, of semigroups with identity and of rings with identity, and the varieties of commutative semigroups with identity and of commutative rings with identity are not solutionally compatible.

Proof. Let $Z$ be the ring of integers and $B=Z[x] /(2 x)$. By identifying the elements of $Z$ with the corresponding residue classes, $B$ becomes an extension of $Z$. The equation

$$
1=2 x
$$

has a solution in the ring or the multiplicative semigroup of the rational numbers and therefore it is solvable over $Z$ in all varieties mentioned in the Theorem. However this equation is not solvable over $B$ because 2 is a zero-divisor in $B$.

\section{References}

G. Grätzer (1971), Lattice Theory (Freeman, San Francisco, 1971).

H. Hule (1970), 'Algebraische Gleichungen über universalen Algebren', Monatsh. Math. 74, 50-55.

B. Jónsson (1956), 'Universal relational systems', Math. Scand. 4, 193-208.

B. Jónsson (1961), 'Sublattices of a free lattice', Canad. J. Math. 13, 256-264.

H. Lausch and W. Nöbauer (1973), Algebra of Polynomials (North Holland, Amsterdam, 1973).

Departamento de Matemático

Universidade de Brasilia

70,000 Brasilia, D.F.

Brazil.

Institut für Mathematik I

Universität für Bildungswissenschaften

A-9010 Klagenfurt

Australia. 